\title{
Induction of protective immune responses against Schistosomiasis haematobium in hamsters and mice using cysteine peptidase-based vaccine
}

\author{
Hatem Tallima ${ }^{1}$ *, John P. Dalton ${ }^{2}$ and Rashika El Ridi ${ }^{1}$ \\ 1 Zoology Department, Faculty of Science, Cairo University, Giza, Egypt \\ ${ }^{2}$ Medical Biology Centre, School of Biological Sciences, Queen's University Belfast, Belfast, UK
}

\section{Edited by:}

Laurent Renia, Agency for Science,

Research and Technology, Singapore

\section{Reviewed by:}

Fabio Trindade Costa, University of Campinas, Brazil

Raymond John Pierce, University of Lille Nord de France, France

\section{*Correspondence:}

Hatem Tallima, Immunology Unit, Zoology Department, Faculty of

Science, Cairo University, Giza, Egypt e-mail: hatemtallima@gmail.com
One of the major lessons we learned from the radiation-attenuated cercariae vaccine studies is that protective immunity against schistosomiasis is dependent on the induction of T helper (Th)1-/Th2-related immune responses. Since most schistosome larval and adultworm-derived molecules used for vaccination uniformly induce a polarized Th1 response, it was essential to include a type 2 immune response-inducing molecule, such as cysteine peptidases, in the vaccine formula. Here, we demonstrate that a single subcutaneous injection of Syrian hamsters with $200 \mu \mathrm{g}$ active papain, $1 \mathrm{~h}$ before percutaneous exposure to 150 cercariae of Schistosoma haematobium, led to highly significant $(P<0.005)$ reduction of $>50 \%$ in worm burden and worm egg counts in intestine. Immunization of hamsters with $20 \mu \mathrm{g}$ recombinant glyceraldehyde 3-phosphate dehydrogenase (rSG3PDH) and $20 \mu \mathrm{g}$ 2-cys peroxiredoxin-derived peptide in a multiple antigen peptide construct (PRX MAP) together with papain ( $20 \mu \mathrm{g} / \mathrm{hamster})$, as adjuvant led to considerable $(64 \%)$ protection against challenge $S$. haematobium infection, similar to the levels reported with irradiated cercariae. Cysteine peptidases-based vaccination was also effective in protecting outbred mice against a percutaneous challenge infection with $S$. haematobium cercariae. In two experiments, a mixture of Schistosoma mansoni cathepsin B1 (SmCB1) and Fasciola hepatica cathepsin L1 (FhCL1) led to highly significant $(P<0.005)$ reduction of $70 \%$ in challenge $S$. haematobium worm burden and $60 \%$ reduction in liver egg counts. Mice vaccinated with SmCB1/FhCL1/rSG3PDH mixture and challenged with S. haematobium cercariae 3 weeks after the second immunization displayed highly significant $(P<0.005)$ reduction of $72 \%$ in challenge worm burden and no eggs in liver of 8-10 mice/group, as compared to unimmunized mice, associated with production of a mixture of type 1- and type 2-related cytokines and antibody responses.

Keywords: Schistosoma haematobium, schistosomiasis vaccine, cysteine peptidases, papain, cathepsins, type 1 and $\mathbf{2}$ immune responses, larval excretory-secretory products

\section{INTRODUCTION}

Schistosomiasis is a debilitating parasitic disease that affects humans in 74 countries, mainly in the Middle East, sub-Saharan Africa, South America, and some regions of the Philippines, China, and Indonesia. Two species, Schistosoma mansoni and Schistosoma haematobium, are responsible for the majority of human infections. As a result of the insensitivity and unreliability of current diagnostic techniques and the paucity of sound epidemiological surveys, it is not clear whether the number of active Schistosoma infections is 209 (1), 230 (2), 252 (3), or 391-587 (4) million people worldwide. People infected with schistosomes react intensely to antigens derived from the huge numbers of parasite eggs that have failed to escape to the exterior via feces (S. mansoni) or urine ( $S$. haematobium), and are trapped in the host tissues. These intense immunological reactions lead to fibrosis and dysfunction of the affected organs, namely liver, gut, and urinary bladder (1-5).
A single anti-schistosome drug, praziquantel (PZQ), is readily available. Despite its low cost and self-limiting side-reactions, the drug has only been offered to less than $13 \%$ of the target population (1). Innumerable persons are left untreated, suffering long-term disabilities and exacerbation of co-infections (1-6). Praziquantel is highly effective in treatment of light and moderate infections. However, in areas of high endemicity and transmission and/or intensive PZQ mass administration, PZQ cure rates are almost negligible [(7) and references therein]. A schistosomiasis vaccine could protect up to $600-780$ million individuals, mostly children, living in endemic regions at risk of the infection. Articles in this topic and elsewhere have duly reported on the history and fate of a number of candidates and potential vaccine antigens, of which very few have shown satisfactory efficacy and none has reached the commercial level (8-10).

One of the main reasons hindering the development of a vaccine against schistosomiasis is the entrenched dogma stating 
protection is dependent on the generation of type 1 immune responses. This belief was based on preponderance of interferongamma $($ IFN- $\gamma)$ released by bronchoalveolar leukocytes, total lung tissue, and lung-draining lymph nodes in radiation-attenuated (RA) cercariae-vaccinated mice $(11,12)$. Lung schistosomuladerived antigens seeping in lung tissues or released from extravasated dying larvae expectedly induce preponderant type 1 immune responses $[(13,14)$ and references therein]. Yet, these immune responses might be irrelevant to parasite attrition, as it must be reiterated healthy schistosomes are exclusively intravascular and may not be directly affected by the immune events in lung alveoli, parenchyma, or draining lymph nodes. More importantly, several studies using knockout mice conclusively demonstrated that the optimal protection in the RA vaccine model is dependent on the induction of both type- 1 and type-2-associated immune responses (15-17).

We have well-learned the lessons of the successful RA vaccine model and thought it is imperative to use type 2-, not type 1inducing cytokines or molecules as adjuvants to the schistosomederived antigens used for vaccination (14). The highly significant $(P<0.0001)$ and reproducible protection against challenge $S$. mansoni worms achieved in mice, immunized with larval antigens derived from excretory-secretory products (ESP), namely recombinant glyceraldehyde 3-phosphate dehydrogenase (rSG3PDH) and 2-cys peroxiredoxin-derived peptide in a multiple antigen peptide construct (PRX MAP) in conjunction with papain, interleukin (IL)-25, IL-33, or thymic stromal lymphopoietin (TSLP), supported our belief. Our proposal was particularly strengthened by the significant $(P<0.02)$ levels of protection obtained following immunization with papain, IL-25, or IL-33 alone $[(18,19)$ and references therein]. Therefore, we felt it was important to examine whether this approach could be applied to S. haematobium, and examined immunological and parasitological parameters in hamsters immunized with papain alone, or papain in conjunction with rSG3PDH and PRX MAP.

Since papain, IL-25, IL-33, or TSLP may not be readily used for human vaccination, we resolved the issue by replacing these type 2-inducing molecules by parasite-derived cysteine peptidase, namely S. mansoni cathepsin B1 (SmCB1). Immunization of outbred mice with $\mathrm{SmCB} 1$ alone generated a polarized type 2 immune response environment that was associated with highly significant $(P<0.0001)$ reduction of $83 \%$ of $S$. mansoni challenge worm burden; this supported our hypothesis stating that S. mansoni larvae will almost all succumb if met by a type 2 cytokine environment (18-21). To further improve the vaccine efficacy, we included another cysteine peptidase, Fasciola hepatica cathepsin L with the aim of inducing the production of anti-cathepsin $\mathrm{L}$ antibodies that would neutralize the $S$. mansoni homologous enzyme and inhibit its function. The highest level of worm burden reduction and decrease in worm egg counts in liver and small intestine of outbred mice were achieved when this peptide formulation was combined with rSG3PDH (22-25). It is important to note that SG3PDH is a larval and adult worm ESP (26), documented to be also associated with the larval surface membrane (22), and to induce polarized type 1 and type 17 immune responses (19).

Therefore, we proposed a novel schistosome cysteine peptidasebased formula that fulfills all requirements for an efficacious vaccine for schistosomiasis $(24,25)$. First, two immunizations are sufficient to induce highly significant $(P<0.0001)$ and highly reproducible (eight experiments) reduction of up to $66 \%$ in $S$. mansoni worm burden and egg counts in host liver and intestine. Second, the vaccine is adjuvant/chemical free, bypassing the insurmountable obstacle of adjuvant use in pre- and clinical trials in humans. Third, vaccine-induced protection is associated with generation of both type 1 and type 2 cytokines-related immune responses. Fourth, the vaccine was entirely safe in outbred mice and did not induce IgE antibodies or any adverse reaction during immunization and after challenge.

To proceed forward with and efficacious vaccine formula against $S$. mansoni, it is important that we demonstrate that these approaches apply to S. haematobium and S. japonicum. Indeed, most vaccine strategies applied to date have not shown crossspecies efficacy. Moreover, vaccine studies in the S. haematobium model are rather rare and, thus, the present study represents an addition to this neglected field. Accordingly, we, herein, investigated whether our vaccine formulation of functional cysteine peptides without the addition of a chemical adjuvant is also effective in protecting mice against a challenge infection with $S$. haematobium.

\section{MATERIALS AND METHODS ETHICS STATEMENT}

All animal experiments were performed following the recommendations of the current edition of the Guide for the Care and Use of Laboratory Animals, Institute of Laboratory Animal Resources, National Research Council, USA, and were approved by the Institutional Animal Care and Use Committee (IACUC) of the Faculty of Science, Cairo University, permit number CUFS F PHY 2114.

\section{ANIMALS AND PARASITES}

Female Syrian hamsters (Mesocricetus auratus) and CD1 mice were raised at the Schistosome Biological Materials Supply Program, Theodore Bilharz Research Institute (SBSP/TBRI), Giza, Egypt, and maintained throughout experimentation at the animal facility of the Zoology Department, Faculty of Science, Cairo University. Cercariae of an Egyptian strain of S. haematobium were obtained from SBSP/TBRI, and used for infection immediately after shedding from Bulinus truncatus snails.

\section{PAPAIN AND IMMUNOGENS}

Papain from Carica papaya (BioChemika $\geq 3$ units/mg) was obtained from BioChemika, and used in an active form or following inactivation by incubation in the presence of $5 \mu \mathrm{M}$ of the irreversible inhibitor of cysteine peptidases, Ltrans-epoxysuccinylleucylamide-(4-guanido)-butane (E-64, Calbiochem, San Diego, CA, USA), as described previously $(24,27)$. Recombinant S. mansoni glyceraldehyde 3-phosphate dehydrogenase (rSG3PDH) was prepared and purified to homogeneity, as described (23) and contained $<0.06$ Endotoxin Units $/ \mathrm{ml}$ as judged by the Pyrogen Gel-Clot Limulus Amebocyte Lysate test. 2-Cys peroxiredoxin (28) (H- ${ }^{104}$ RKQEISKAYGVFDE EDGNA ${ }^{122}$ $\mathrm{OH}$ )-derived peptide, showing lowest homology to the murine counterpart, was synthesized as a MAP (tetra branched multiple antigen peptide) construct (PRX MAP) and purified at AnaSpec 
Inc. (San Jose, CA, USA). Functionally active S. mansoni cathepsin B1 (SmCB1) and F. hepatica cathepsin L1 (FhCL1) were prepared as described $(24,29)$.

These $S$. mansoni-derived molecules were used for vaccination of hamsters and mice against infection with S. haematobium, as they are remarkably conserved across S. mansoni and S. haematobium. S. mansoni cathepsin B1 and SG3PDH show 94-96\% identities at the amino acid level with the corresponding enzyme of S. haematobium (GI:68596858 and GI:685936895, respectively). S. mansoni 2-Cys peroxiredoxin (PRX)-derived peptide used in the MAP construct shows $84 \%$ identities and $89 \%$ similarity with the corresponding peptide of S. haematobium PRX (GI:685965340). F. hepatica cathepsin L was readily recognized by outbred mice infected with S. mansoni.

\section{INJECTION, INFECTION, AND ANALYSES IN HAMSTERS}

Hamsters (10 per group) were injected subcutaneously (sc) into two sites with $200 \mu \mathrm{g}$ active papain. One hour later, injected and untreated hamsters were anesthetized, abdomen-shaved, and then exposed percutaneously using the ring method (30) to $150 \mathrm{~S}$. haematobium cercariae.

Hamsters ( 10 or 11 per group) were immunized, twice at 3 weeks interval, sc on one side with $20 \mu \mathrm{g}$ active or E-64-inactivated papain, and intramuscularly on the other side with $20 \mu \mathrm{g}$ rSG3PDH and $20 \mu \mathrm{g}$ PRX MAP. Four weeks later, naïve and immunized hamsters were exposed to 120 cercariae of S. haematobium as described above.

Serum was recovered from three hamsters per group per experiment on day 14 post infection. Sera were individually assessed by enzyme-linked immunosorbent assay (ELISA) for antibody binding to soluble $S$. haematobium adult worm antigen (SAWA, $1.0 \mu \mathrm{g} /$ well) prepared as described (31), rSG3PDH (250 ng/well), and PRX MAP (1.0 $\mu \mathrm{g} /$ well). Alkaline phosphatase (AKP)-labeled anti-hamster IgG $(\mathrm{H}+\mathrm{L})$ conjugate (Kirkegaard and Perry Laboratories, Gaithersburg, MD, USA) was diluted 1:1000. For each experiment, antibody isotypes of individual sera, diluted 1:50, for each hamster group were determined using biotin-labeled monoclonal antibodies to hamster IgG classes, IgG1, and IgG2 (Pharmingen, San Diego, CA, USA), and AKP-labeled streptavidin from Promega (Madison, WI, USA).

Worm burden and liver and intestine worm egg load in individual hamsters (6-8 per group) were evaluated 12 weeks after challenge infection $(19,24)$. Mean values \pm SE for each group were calculated. Percent change was evaluated by the formula: $\%$ change $=$ mean number in infected controls - mean number in infected, treated mice/mean number in infected controls $\times 100$.

\section{IMMUNIZATIONS AND INFECTIONS IN MICE}

In two experiments, mice (12-13 per group) were immunized sc at the base of the tail, twice with a 3-week interval with $10 \mu \mathrm{g}$ SmCB1 and $10 \mu \mathrm{g}$ FhCL1 alone or combined with rSG3PDH (10 $\mu \mathrm{g} /$ mouse). Three weeks after the second injection, untreated and immunized mice were infected percutaneously via whole body exposure to $100 \pm 5$ viable cercariae of S. haematobium. Spleen cells and serum were recovered from 2-3 mice per group per experiment on day 8 post infection. Worm burden and liver worm egg load were assessed in mice 12 weeks after the challenge infection as described for hamsters.

\section{CYTOKINE AND HUMORAL RESPONSES}

Spleen cells (SC) were harvested on day 8 after infection with $S$. haematobium cercariae, and cultured with 0 or $5 \mu \mathrm{g} / \mathrm{ml}$ immunogen as described $(19,24)$. At 48 and $72 \mathrm{~h}$ of incubation, cultured SC were thawed and frozen for release of intracellular cytokines, and supernatants stored at $-76^{\circ} \mathrm{C}$ until assayed by capture ELISA for levels of IL-4, IL-5, IL-17A, IFN- $\gamma$ (ELISA MAX ${ }^{\mathrm{TM}}$ Set, BioLegend), and IL-13 (DuoSet ELISA Development System, R\&D Systems Europe), following the manufacturer's instructions.

Sera were obtained from unimmunized and immunized mice 8 days following infection with cercariae of S. haematobium, and individually assessed by ELISA for humoral antibody titer reactivity to SAWA (1.0 $\mu \mathrm{g} /$ well) and SmCB1 (250 ng per well). Antibody isotypes in mouse sera (1:200 dilution) were analyzed using rat alkaline phosphatase-conjugated monoclonal antibodies to various mouse IgG classes (Pharmingen) and biotin-labeled monoclonal antibody to mouse IgA, and $\operatorname{IgE}$ (BioLegend) with sera diluted 1:25 $(19,24)$.

\section{DATA ANALYSIS AND STATISTICS}

All values were tested for normality. Mann-Whitney test was used to analyze the statistical significance of differences between experimental and control values and considered significant at $P<0.05$.

\section{RESULTS}

\section{EFFECT OF PRE-TREATMENT WITH THE CYSTEINE PEPTIDASE, PAPAIN ON HAMSTER HUMORAL IMMUNE RESPONSES, AND RESISTANCE TO S. HAEMATOBIUM}

Hamster humoral antibody binding to soluble adult worm antigens (SAWA) 14 days after exposure to 150 cercariae of S. haematobium was negligible, and was not significantly enhanced by pre-infection treatment with active papain (data not shown). Yet, a single papain injection $1 \mathrm{~h}$ before hamster exposure to $S$. haematobium cercariae led to highly significant $(P<0.005)$ decrease in total worm burden of $55 \%$. The decrease in worm burden was observed for both male and female worms (Table 1). Hamster pre-treatment with active papain before infection did not induce a significant decrease in worm egg counts in liver. However, the decrease in intestine egg counts was highly significant $(P<0.002)$, reaching $78.1 \%$ (Table $\mathbf{1}$ ).

\section{EFFECTS OF THE CYSTEINE PEPTIDASE, PAPAIN AS ADJUVANT ON HAMSTER HUMORAL IMMUNE RESPONSES, AND RESISTANCE TO $S$. HAEMATOBIUM}

Hamsters immunized with rSG3PDH and PRX MAP in conjunction with inactive or active papain were challenged with $120 \mathrm{~S}$. haematobium cercariae 4 weeks later, and tested for serum antibody binding to SAWA and to the immunogens 14 days after infection. Highest antibody binding to SAWA was observed in hosts immunized with the vaccine in conjunction with active papain. The antibody binding to rSG3PDH and PRX MAP, 6 weeks after the boost immunization, is evidence for memory response to 
the immunogens, and was again highest in hamsters immunized with the vaccine and active papain as adjuvant (Figure 1). No immunogen-specific IgG1 antibodies were detected, while about $10 \%$ of the bound antibodies were of the IgG2 isotype (data not shown).

Active papain used as an adjuvant to rSG3PDH/PRX MAP vaccination of hamsters against $S$. haematobium led to highly significant $(P=0.0007)$ decrease of $64 \%$ in total worm burden. The

Table 1 | Effect of papain pre-treatment on parasitological parameters of $\boldsymbol{S}$. haematobium-infected hamsters.

\begin{tabular}{lcc}
\hline Parameter & Infected controls & Active papain \\
\hline Total worm burden & & \\
Mean \pm SE & $48.8 \pm 2.6$ & $22.1 \pm 0.9$ \\
$P$ value (reduction \%) & & $<0.005(54.7)$ \\
Male worm burden & & $13.3 \pm 0.8$ \\
Mean \pm SE & $30.5 \pm 2.6$ & $<0.005(56.3)$ \\
$P$ value (reduction \%) & & \\
Female worm burden & & $8.5 \pm 0.7$ \\
Mean \pm SE & $17.8 \pm 1.2$ & $<0.005(52.2)$ \\
$P$ value (reduction \%) & & \\
Liver egg counts & & $60000 \pm 5400$ \\
Mean \pm SE & $70450 \pm 1646$ & Not significant \\
$P$ value (reduction \%) & & \\
Intestine egg counts & & $3620 \pm 616$ \\
Mean \pm SE & $16564 \pm 2210$ & $<.002(78.4)$ \\
$P$ value (reduction \%) & & \\
\hline
\end{tabular}

Parasitological parameters were assessed 12 weeks after infection of untreated (infection controls), or active papain-injected hamsters (7 hamsters per group). Two-tailed $P$ value as assessed by the Mann-Whitney test. Reduction $\%=$ mean number of untreated hamsters - mean number of papain treated hamsters/mean number in untreated hamsters $\times 100$. decrease in worm burden was observed for both male and female worms. Inactivation of papain with E-64 almost completely eliminated its protective effect (Table 2; Figure 2). Importantly, hamster immunized with rSG3PDH/PRX MAP and active papain as adjuvant showed significant decrease in challenge $S$. haematobium worm egg counts in both liver $(32.8 \%, P<0.05)$ and intestine $(59.4 \%, P<0.01)$ (Table 2; Figure 2).

\section{CYSTEINE PEPTIDASE-BASED VACCINE AGAINST MURINE S. HAEMATOBIUM}

In two experiments, 9 out of 9 untreated/infected mice had 46 worms and 950-2400 eggs in liver. Vaccination with SmCB1/ FhCL1 mixture led to highly significant $(P<0.005)$ reduction of $70 \%$ in challenge $S$. haematobium worm burden and $60 \%$ reduction in liver egg counts, as 5 out of 10 mice had 1-2 worms and 3 out of 10 mice showed 850-2400 eggs in liver. Mice vaccinated with SmCB1/FhCL1 and rSG3PDH displayed highly significant $(P<0.005)$ reduction of $72 \%$ in challenge worm burden and no eggs in liver of $8-10$ mice/group, as compared to unimmunized mice.

Spleen cells obtained from unimmunized and immunized mice 8 days following $S$. haematobium infection, and stimulated in vitro with cysteine peptidase or rSG3PDH produced higher levels of IL-4, IL-5, IL-13, and also IL-17 and IFN- $\gamma$, compared to naïve and unimmunized infected mice, implying that cysteine peptidasebased protection against $S$. haematobium was associated with a mixture of type 1, type 2, and type 17 cytokines (Figure 3 ).

Serum antibody titer and isotype responses to SAWA and SmCB1 in unimmunized and immunized mice, 8 days after infection with S. haematobium are shown in Figure 4. Antibody responses to SAWA were observed only in mice immunized with rSG3PDH in conjunction with SmCB1 and FhCL1, and included IgG1, IgG2a, IgG2b, and IgA antibodies. No IgE antibodies were detected in sera diluted 1:25 (Figures 4A,B). High antibody responses to SmCB1 were observed in mice immunized with the cysteine peptidases and consisted predominantly of IgG1 antibodies. Serum antibody responses to SmCB1 were highest in

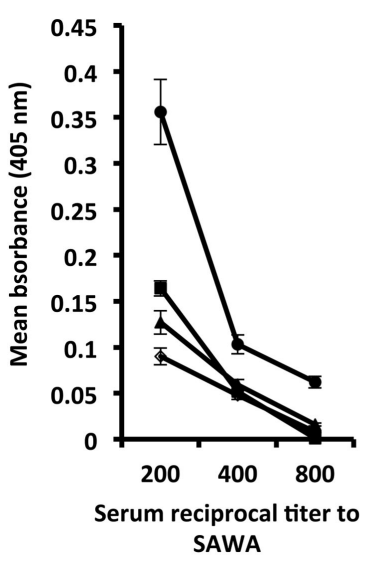

FIGURE 1 | Effect of combining rSG3PDH/PRX MAP vaccine with active papain on humoral responses of 14 day

$\boldsymbol{S}$. haematobium-infected hamsters. Each point represents mean ELISA absorbance $(405 \mathrm{~nm}$ ) of sera from three individual naïve (o),
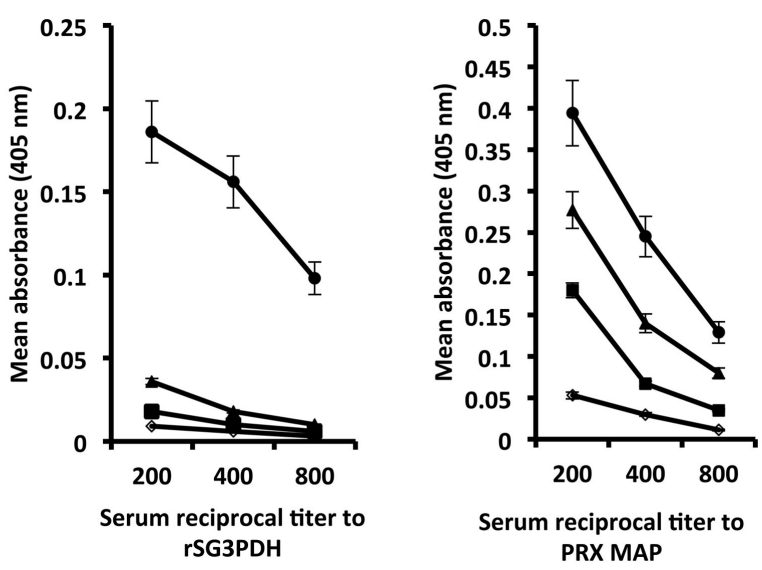

infected control (घ) hamsters, and hamsters immunized with inactive $(\boldsymbol{\Delta})$ and active $(\bullet)$ papain in conjunction with rSG3PDH and PRX MAP, tested in duplicate, and the horizontal bars depict the SE around the mean. 
mice immunized with the cysteine peptidases in conjunction with rSG3PDH, and included IgG1, IgG2a, IgG2b, and IgA antibodies (Figures 4C,D).

Table 2 | Effect of combining rSG3PDH/PRX MAP vaccine with inactive or active papain on parasitological parameters of $S$.

haematobium-infected hamsters.

\begin{tabular}{lccc}
\hline Parameter & $\begin{array}{c}\text { Infected } \\
\text { controls }\end{array}$ & $\begin{array}{c}\text { Inactive } \\
\text { papain/Ag mix }\end{array}$ & $\begin{array}{c}\text { Active } \\
\text { papain/Ag mix }\end{array}$ \\
\hline Total worm burden & & & \\
Mean \pm SE & $25.5 \pm 2.0$ & $18.7 \pm 2.4$ & $9.3 \pm 2.8$ \\
$P$ value (reduction \%) & NS & $0.0007(63.5)$ \\
Male worm burden & & & \\
Mean \pm SE & $15.5 \pm 1.1$ & $11.8 \pm 1.9$ & $5.1 \pm 0.6$ \\
$P$ value (reduction \%) & & NS & $0.0007(67.1)$ \\
Female worm burden & & & \\
Mean \pm SE & $10.0 \pm 0.9$ & $6.8 \pm 0.7$ & $4.2 \pm 0.4$ \\
$P$ value (reduction \%) & & $0.037(32.0)$ & $0.0007(58.0)$ \\
Liver egg counts & & & \\
Mean \pm SE & $19833 \pm 2438$ & $14714 \pm 937$ & $13312 \pm 1505$ \\
$P$ value (reduction \%) & & $\mathrm{NS}$ & $0.0481(32.8)$ \\
Intestine egg counts & & & \\
Mean \pm SE & $5333 \pm 840$ & $4028 \pm 566$ & $2162 \pm 301$ \\
$P$ value (reduction \%) & & $\mathrm{NS}$ & $0.0067(59.4)$ \\
\hline
\end{tabular}

Ag mix $=r S G 3 P D H+P R X$ MAP. NS = not significant, as assessed by the MannWhitney test (two-tailed $P$ value). Reduction $\%=$ mean number in unimmunized hamsters-mean number in papain/Ag mix immunized hamsters/mean number in unimmunized hamsters $\times 100$.

\section{DISCUSSION}

We have proposed that to achieve significant attrition of schistosomes during their journey in the lung blood capillaries and liver sinusoids, eosinophils and basophils must be recruited to the circulation and activated by type 2 cytokines, such as IL-4, IL-5, and IL-13, or via binding antibody-ESP complexes in the vicinity of the larvae (18-21). Worms-derived antigens induce preponderant type 1- and type 17- and limited or no type 2related cytokines and antibodies. Accordingly, we have proposed that deviation of the host immune system toward the type 2 axis, via administering type 2 immune responses-inducing molecules, such as the cysteine peptidase, papain (32-34) before infection will lead to considerable decrease in worm burden, as compared to uninjected animals. In four consecutive experiments, $50 \mu \mathrm{g}$ papain injected sc in outbred mice, $1 \mathrm{~h}$ before exposure to 125 cercariae of $S$. mansoni consistently, and reproducibly elicited highly significant $(P<0.0001)$ reduction in worm burden of $70 \% \pm 3$. The reduction was also highly significant for decrease in worm liver $(P<0.0001)$ and small intestine $(P<0.001)$ egg counts but only of approximately $50 \%$ (14).

Here, our studies with peptidases were extended to determine the validity of this approach for protection in the hamster model of S. haematobium (Table 1). We found that papain treatment prior to challenge infection caused a reduction in S. haematobium worm burden in hamsters which was not associated with significant humoral responses. We are currently exploring the molecular mechanism by which pre-treatment with active papain leads to such highly significant $(P<0.005)$ decrease in total, male and female worm burden. Second, while the reductions in intestine egg counts were considerable in papain-injected hamsters, there was no decrease in liver egg counts; this could suggest elevated fecundity in the surviving worms, perhaps due to increased levels

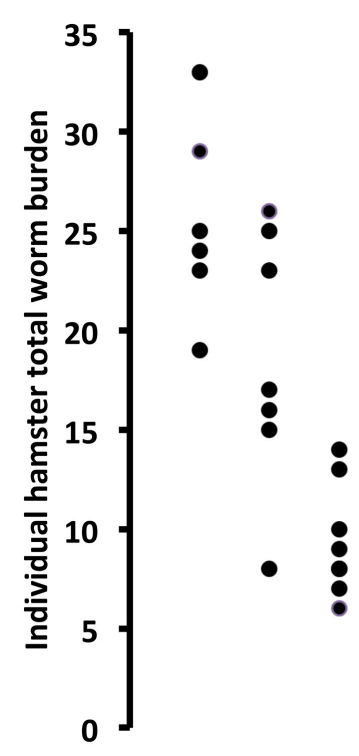

FIGURE 2 | Effect of combining rSG3PDH/PRX MAP vaccine with inactive (middle column in each panel) or active (right column in each panel) papain on parasitological parameters of $S$. haematobium-infected

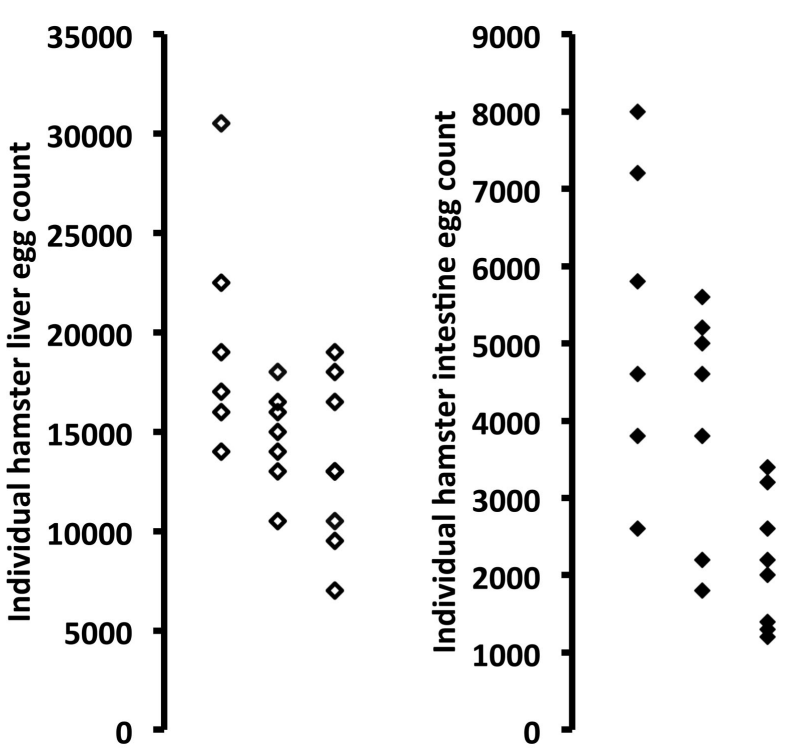

hamsters, as compared to unimmunized hamsters (left column in each panel). Each point represents parasitological parameter in individual hamsters, 12 weeks after infection with $S$. haematobium cercariae. 


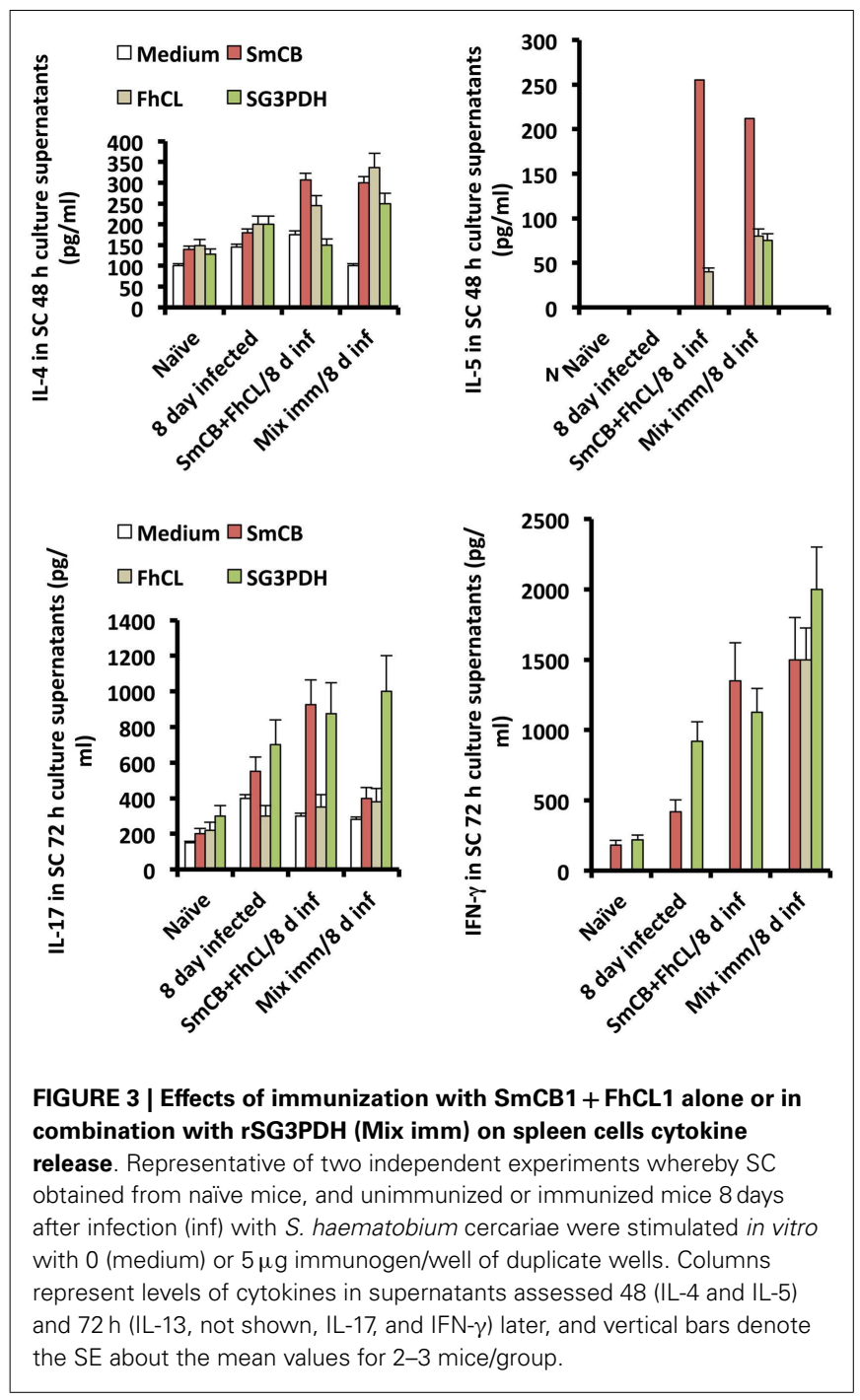

of type 2 cytokines which has been reported to correlate with schistosome increased egg production $(24,35)$.

Vaccination of hamsters with our candidate vaccine mixture, rSG3PDH, and PRX MAP, in conjunction with papain led to highly significant $(P=0.0007)$ decrease of $64 \%$ in $S$. haematobium challenge worm burden provided using active not E-64inactivated papain. These findings extend and confirm results recently obtained in mice immunized with the antigen mixture in conjunction with active papain and challenged with S. mansoni. It is of importance to note that active, but not inactivated papain, helped to generate immunogens-specific humoral antibody response that appeared to be essential for protection and decrease in worm egg load in liver. These findings suggest that the key to papain-mediated protective effect may be induction of active enzyme activity-dependent long-lived antibody-secreting cells, similarly to the proteases, natterins from the venom of Thalassophryne nattereri fish $(36,37)$.

Since papain derived from the plant Carica papaya could not be used for human vaccination, we sought to replace this by the $S$. mansoni cysteine peptidase, SmCB1, and the F. hepatica

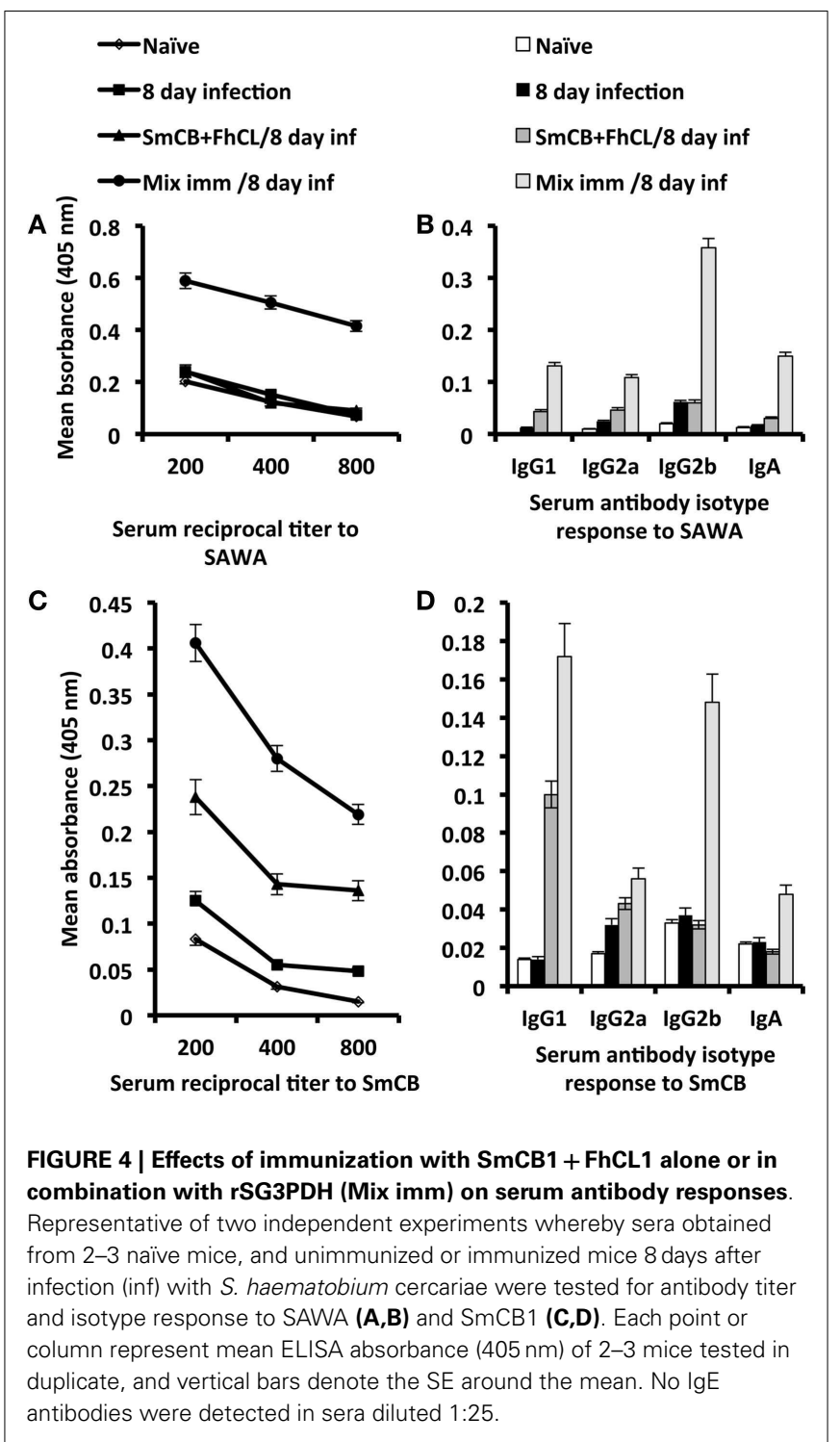

cathepsin L, both members of the papain-like peptidase family. As reported previously, immunization of outbred mice with SmCB1 + FhCL1 + rSG3PDH elicited highly significant $(P<0.0001)$ decrease of about $66 \%$ in challenge $S$. mansoni worm burden and worm egg counts in liver and intestine, distinctly higher than for SmCB1 and FhCL1, without rSG3PDH $(24,25)$. These results were reproduced here since by demonstrating protection of mice against challenge $S$. haematobium with similar formulations. Protection against murine S. haematobium appeared to be associated with induction of Th1, Th17, and Th2 cytokines and antibody responses, corroborating our suggestion that the generation of type 2-related immune responses is important in the design of an effective schistosomiasis vaccine (18-21, 24, 25).

The cysteine peptidases used in the study are purified as zymogens, which are stabilized by their propeptide segments. The schistosome and fasciola peptidases have been shown previously by Dalton and colleagues $(38-41)$ to be extremely stable and not readily susceptible to breakdown. Jilkova et al. (42) recently 
described how the propeptide of SmCB1 can stabilize the enzyme and resist auto-processing, and suggested that the enzyme can be activated when delivered in vivo by tissue glycoaminoglycans. Therefore, it is possible that following injection of the cysteine proteases, these become activated to mature enzymes by interaction with glycoaminoglycans under the skin.

Concerns may be raised for the future use of cysteine peptidases for vaccination of humans because of their potential to induce IgE antibodies. However, the cysteine peptidases used in the vaccine formula consistently failed to elicit production of IgE in mice despite booster immunizations with SmCB1 and FhCL1 alone or combined (this study and 24). Additionally, serum antibodies of S. mansoni-infected humans that bound to SmCB1 and FhCL1 were found to be essentially of the $\operatorname{IgG}$ and $\operatorname{IgA}$ isotype $(24,43$, 44). Nonetheless, helminth cysteine peptidases-induced IgE may be irrelevant for children in rural areas of the developing world, to whom the vaccine is intended, as these usually harbor other parasites that stimulate IgE antibodies of diverse specificities, thus precluding harmful hypersensitive reactions. Pre-clinical studies in healthy volunteers must be performed before any positive or negative conclusion can be drawn regarding cysteine peptidase-based vaccine implementation in humans.

\section{ACKNOWLEDGMENTS}

Thanks are extended to Mr. Abdel Badih Foda for excellent and dedicated care of the experimental hamsters and mice. We acknowledge the contribution of the reviewers in improving the quality of the manuscript presentation.

\section{REFERENCES}

1. World Health Organization. Schistosomiasis: population requiring preventive chemotherapy and number of people treated in 2010. Wkly Epidemiol Rec (2012) 87(4):37-44.

2. Colley DG, Bustinduy AL, Secor WE, King CH. Human schistosomiasis. Lancet (2014) 383(9936):2253-64. doi:10.1016/S0140-6736(13)61949-2

3. Hotez PJ, Alvarado M, Basáñez MG, Bolliger I, Bourne R, Boussinesq M, et al. The global burden of disease study 2010: interpretation and implications for the neglected tropical diseases. PLoS Negl Trop Dis (2014) 8(7):e2865. doi:10.1371/journal.pntd.0002865

4. King CH. Parasites and poverty: the case of schistosomiasis. Acta Trop (2010) 113(2):95-104. doi:10.1016/j.actatropica.2009.11.012

5. King $\mathrm{CH}$, Dangerfield-Cha $\mathrm{M}$. The unacknowledged impact of chronic schistosomiasis. Chronic Illn (2008) 4(1):65-79. doi:10.1177/1742395307084407

6. King $\mathrm{CH}$, Bertino AM. Asymmetries of poverty: why global burden of disease valuations underestimate the burden of neglected tropical diseases. PLoS Negl Trop Dis (2008) 2(3):e209. doi:10.1371/journal.pntd.0000209

7. Utzinger J, N'Goran EK, N’Dri A, Lengeler C, Tanner M. Efficacy of praziquantel against Schistosoma mansoni with particular consideration for intensity of infection. Trop Med Int Health (2000) 5(11):771-8. doi:10.1046/j.1365-3156. 2000.00646.x

8. Tendler M, Simpson AJ. The biotechnology-value chain: development of Sm14 as a schistosomiasis vaccine. Acta Trop (2008) 108(2-3):263-6. doi:10.1016/j. actatropica.2008.09.002

9. McManus DP, Loukas A. Current status of vaccines for schistosomiasis. Clin Microbiol Rev (2008) 21(1):225-42. doi:10.1128/CMR.00046-07

10. Figueiredo BC-P, Ricii ND, Assis NRG, Morais SB, Fonseca CT, Oliveira SC. Kicking in the guts: Schistosoma mansoni digestive tract proteins are potential candidates for vaccine development. Front Immunol (2015) 6:22. doi:10.3389/ fimmu.2015.00022

11. Wynn TA, Oswald IP, Eltoum IA, Caspar P, Lowenstein CJ, Lewis FA, et al. Elevated expression of Thl cytokines and nitric oxide synthase in the lungs of vaccinated mice after challenge infection with Schistosoma mansoni. J Immunol (1994) 153(11):5200-9.
12. Wilson RA, Coulson PS, Betts C, Dowling MA, Smythies LE. Impaired immunity and altered pulmonary responses in mice with a disrupted interferon-gamma receptor gene exposed to the irradiated Schistosoma mansoni vaccine. Immunology (1996) 87(2):275-82. doi:10.1046/j.1365-2567.1996. 465550.x

13. Mountford AP, Harrop R, Wilson RA. Antigens derived from lung-stage larvae of Schistosoma mansoni are efficient stimulators of proliferation and gamma interferon secretion by lymphocytes from mice vaccinated with attenuated larvae. Infect Immun (1995) 63(5):1980-6.

14. El Ridi R, Tallima $H$. Why the radiation-attenuated cercarial immunization studies failed to guide the road for an effective schistosomiasis vaccine: a review. JAdv Res (2015) (in press).

15. Anderson S, Shires VL, Wilson RA, Mountford AP. In the absence of IL-12, the induction of Th1-mediated protective immunity by the attenuated schistosome vaccine is impaired, revealing an alternative pathway with Th2-type characteristics. Eur J Immunol (1998) 28(9):2827-38. doi:10.1002/(SICI)15214141(199809)28:09<2827::AID-IMMU2827>3.0.CO;2-K

16. Hoffmann KF, James SL, Cheever AW, Wynn TA. Studies with double cytokinedeficient mice reveal that highly polarized Th1- and Th2-type cytokine and antibody responses contribute equally to vaccine-induced immunity to Schistosoma mansoni. J Immunol (1999) 163(2):927-38.

17. Wynn TA, Hoffmann KF. Defining a schistosomiasis vaccination strategy - is it really Th1 versus Th2? Parasitol Today (2000) 16(11):497-501. doi:10.1016/ S0169-4758(00)01788-9

18. El Ridi R, Tallima H. Adjuvant selection for vaccination against murine schistosomiasis. Scand J Immunol (2012) 76(6):552-8. doi:10.1111/j.1365-3083.2012. 02768.x

19. El Ridi R, Tallima H. Vaccine-induced protection against murine schistosomiasis mansoni with larval excretory-secretory antigens and papain or type-2 cytokines. J Parasitol (2013) 99(2):194-202. doi:10.1645/GE-3186.1

20. El Ridi R, Tallima H. Solving the riddle of the lung-stage schistosomula paved the way to a novel remedy and an efficacious vaccine for schistosomiasis. In: El Ridi R, editor. Parasitic Diseases - Schistosomiasis. Croatia: InTech (2013). p. 197-202. Available from: http://www.intechopen.com/books/parasiticdiseases-schistosomiasis/solving-the-riddle-of-the-lung-stage-schistosomulapaved-the-way-to-a-novel-remedy-and-an-efficaciou

21. El Ridi RAF, Tallima HA-M. Novel therapeutic and prevention approaches for schistosomiasis: review. J Adv Res (2013) 4(5):467-78. doi:10.1016/j.jare.2012. 05.002

22. Goudot-Crozel V, Caillol D, Djabali M, Dessein AJ. The major parasite surface antigen associated with human resistance to schistosomiasis is a $37-\mathrm{kD}$ glyceraldehyde-3P-dehydrogenase. J Exp Med (1989) 170(6):2065-80. doi:10. 1084/jem.170.6.2065

23. El Ridi R, Shoemaker CB, Farouk F, El Sherif NH, Afifi A. Human T- and Bcell responses to Schistosoma mansoni recombinant glyceraldehyde 3-phosphate dehydrogenase correlate with resistance to reinfection with S. mansoni or Schistosoma haematobium after chemotherapy. Infect Immun (2001) 69(1):237-44. doi:10.1128/IAI.69.1.237-244.2001

24. El Ridi R, Tallima H, Selim S, Donnelly S, Cotton S, Gonzales Santana B, et al. Cysteine peptidases as schistosomiasis vaccines with inbuilt adjuvanticity. PLoS One (2014) 9(1):e85401. doi:10.1371/journal.pone.0085401

25. El Ridi R, Tallima H, Dalton JP, Donnelly S. Induction of protective immune responses against schistosomiasis using functionally active cysteine peptidases. Front Genet (2014) 5:119. doi:10.3389/fgene.2014.00119

26. Harrop R, Coulson PS, Wilson RA. Characterization, cloning and immunogenicity of antigens released by lung-stage larvae of Schistosoma mansoni. Parasitology (1999) 118(Pt 6):583-94. doi:10.1017/S003118209900431X

27. Dvorák J, Mashiyama ST, Braschi S, Sajid M, Knudsen GM, Hansell E, et al. Differential use of protease families for invasion by schistosome cercariae. Biochimie (2008) 90(2):345-58. doi:10.1016/j.biochi.2007.08.013

28. Donnelly S, Stack CM, O’Neill SM, Sayed AA, Williams DL, Dalton JP. Helminth 2-Cys peroxiredoxin drives Th2 responses through a mechanism involving alternatively activated macrophages. FASEB J (2008) 22(11):4022-32. doi:10.1096/fj.08-106278

29. Collins PR, Stack CM, O’Neill SM, Doyle S, Ryan T, Brennan GP, et al. Cathepsin L1, the major protease involved in liver fluke (Fasciola hepatica) virulence: propetide cleavage sites and autoactivation of the zymogen secreted from gastrodermal cells. J Biol Chem (2004) 279(17):17038-46. doi:10.1074/jbc. M308831200 
30. Vilar MM, Pinto RM. Reappraisal of experimental infections with cercariae and schistosomula of a Brazilian strain of Schistosoma mansoni in mice. Braz J Biol (2005) 65(4):729-33. doi:10.1590/S1519-69842005000400020

31. El Ridi R, Farouk F, Sherif M, Al-Sherbiny M, Osman A, El Gengehi N, et al. T and $\mathrm{B}$ cell reactivity to a $42-\mathrm{kDa}$ protein is associated with human resistance to both schistosomiasis mansoni and haematobium. JInfect Dis (1998) 177(5):1364-72. doi:10.1086/515274

32. Rosenstein RK, Bezbradica JS, Yu S, Medzhitov R. Signaling pathways activated by a protease allergen in basophils. Proc Natl Acad Sci U S A (2014) 111(46):E4963-71. doi:10.1073/pnas.1418959111

33. Dwyer DF, Woodruff MC, Carroll MC, Austen KF, Gurish MF. B cells regulate $\mathrm{CD} 4+\mathrm{T}$ cell responses to papain following B cell receptor-independent papain uptake. J Immunol (2014) 193(2):529-39. doi:10.4049/jimmunol.1303247

34. Motomura Y, Morita H, Moro K, Nakae S, Artis D, Endo TA, et al. Basophilderived interleukin- 4 controls the function of natural helper cells, a member of ILC2s, in lung inflammation. Immunity (2014) 40(5):758-71. doi:10.1016/j. immuni.2014.04.013

35. Wynn TA. Immune deviation as a strategy for schistosomiasis vaccines designed to prevent infection and egg-induced immunopathology. Microbes Infect (1999) 1(7):525-34. doi:10.1016/S1286-4579(99)80092-6

36. Grund LZ, Komegae EN, Lopes-Ferreira M, Lima C. IL-5 and IL-17A are critical for the chronic IgE response and differentiation of long-lived antibody-secreting cells in inflamed tissues. Cytokine (2012) 59(2):335-51. doi:10.1016/j.cyto.2012. 04.045

37. Komegae EN, Grund LZ, Lopes-Ferreira M, Lima C. The longevity of Th2 humoral response induced by proteases natterins requires the participation of long-lasting innate-like B cells and plasma cells in spleen. PLoS One (2013) 8(6):e67135. doi:10.1371/journal.pone.0067135

38. Dalton JP, Clough KA, Jones MK, Brindley PJ. Characterization of the cathepsin-like cysteine proteinases of Schistosoma mansoni. Infect Immun (1996) 64(4):1328-34.

39. Roche L, Dowd AJ, Tort J, McGonigle S, MacSweeney A, Curley GP, et al. Functional expression of Fasciola hepatica cathepsin L1 in Saccharomyces cerevisiae. Eur J Biochem (1997) 245(2):373-80. doi:10.1111/j.1432-1033.1997.t011-00373.x
40. Dalton JP, Neill SO, Stack C, Collins P, Walshe A, Sekiya M, et al. Fasciola hepatica cathepsin L-like proteases: biology, function, and potential in the development of first generation liver fluke vaccines. Int J Parasitol (2003) 33(11):1173-81. doi:10.1016/S0020-7519(03)00171-1

41. Dalton JP, Dvorak J. Activating the cathepsin B1 of a parasite: a major route with alternative pathways? Structure (2014) 22(12):1696-8. doi:10.1016/j.str. 2014.11.003

42. Jílková A, Horn $M$, Rezáčová $\mathrm{P}$, Marešová L, Fajtová $\mathrm{P}$, Brynda J, et al. Activation route of the Schistosoma mansoni cathepsin B1 drug target: structural map with a glycosaminoglycan switch. Structure (2014) 22(12):1786-98. doi:10.1016/j.str.2014.09.015

43. Chappell CL, Dresden MH, Gryseels B, Deelder AM. Antibody response to Schistosoma mansoni adult worm cysteine proteinases in infected individuals. Am J Trop Med Hyg (1990) 42(4):335-41.

44. de Oliveira Fraga LA, Torrero MN, Tocheva AS, Mitre E, Davies SJ. Induction of type 2 responses by schistosome worms during prepatent infection. J Infect Dis (2010) 201(3):464-72. doi:10.1086/649841

Conflict of Interest Statement: The authors declare that the research was conducted in the absence of any commercial or financial relationships that could be construed as a potential conflict of interest.

Received: 10 December 2014; accepted: 10 March 2015; published online: 23 March 2015.

Citation: Tallima H, Dalton JP and El Ridi R (2015) Induction of protective immune responses against Schistosomiasis haematobium in hamsters and mice using cysteine peptidase-based vaccine. Front. Immunol. 6:130. doi: 10.3389/fimmu.2015.00130

This article was submitted to Immunotherapies and Vaccines, a section of the journal Frontiers in Immunology.

Copyright $(2015$ Tallima, Dalton and El Ridi. This is an open-access article distributed under the terms of the Creative Commons Attribution License (CC BY). The use, distribution or reproduction in other forums is permitted, provided the original author(s) or licensor are credited and that the original publication in this journal is cited, in accordance with accepted academic practice. No use, distribution or reproduction is permitted which does not comply with these terms. 